\title{
Incubation Time and Cavitation Erosion Rate of Work-Hardening Materials
}

\author{
Jean-Pierre Franc LEGI, BP 53, 38041 Grenoble Cedex 9, France, e-mail: jean-pierre.franc@hmg.inpg.fr
}

A phenomenological analysis of the cavitation erosion process of ductile materials is proposed. On the material side, the main parameters are the thickness of the hardened layer together with the conventional yield strength and ultimate strength. On the fluid side, the erosive potential of the cavitating flow is described in a simplified way using three integral parameters: rate, mean amplitude, and mean size of hydrodynamic impact loads. Explicit equations are derived for the computation of the incubation time and the steady-state erosion rate. They point out two characteristic scales. The time scale, which is relevant to the erosion phenomenon, is the covering time - the time necessary for the impacts to cover the material surface-whereas the pertinent length scale for ductile materials is the thickness of the hardened layer. The incubation time is proportional to the covering time with a multiplicative factor, which strongly depends on flow aggressiveness in terms of the mean amplitude of impact loads. As for the erosion rate under steady-state conditions, it is scaled by the ratio of the thickness of hardened layers to the covering time with an additional dependence on flow aggressiveness, too. The approach is sup-ported by erosion tests conducted in a cavitation tunnel at a velocity of $65 \mathrm{~m} / \mathrm{s}$ on stainless steel $316 \mathrm{~L}$. Flow aggressiveness is inferred from pitting tests. The same model of material response that was used for mass loss prediction is applied to derive the original hydrodynamic impact loads due to bubble collapses from the geometric features of the pits. Long duration tests are performed in order to determine experimentally the incubation time and the mean depth of penetration rate and to validate the theoretical approach.

\section{Introduction}

Cavitating flows are characterized by the development of relatively large vapor structures, which usually break up into smaller ones. For instance, a sheet cavity attached to the leading edge of a blade generally splits into many tiny bubbles in its closure region. It may also shed more or less regularly large clouds made of a myriad of small bubbles. If the overall features of the flow depend mainly on the global extent of cavitation, erosion is essentially caused by the individual collapses of small scale vapor structures.

The rate of production of such structures has been the subject of several investigations. Pereira et al. [1] using a tomographic technique could measure the rate of production of small scale vapor structures by a leading edge cavity together with their volume. They showed that the production rate is ruled by a Strouhal-like law, i.e., that the shedding frequency of small scale structures is inversely proportional to their size. The smaller the structures, the larger the production rate. Such a relationship is qualitatively confirmed by pitting tests that show that pit density generally increases when pit size decreases (see, e.g., Fig. 12 of the present work). This comparison assumes that pit size is correlated with bubble size, which seems physically reasonable although still open to discussion. A Strouhal similarity law for the prediction of the production rate of small scale structures was also proposed by Lecoffre et al. [2] following the work of Kato [3] as the basis for analyzing the effects of velocity and length scale on cavitation damage. In the scenario they propose for a quantitative prediction of cavitation erosion, Kato et al. [4] also addressed the problem of the breakup of a sheet cavity into small vapor structures. To quantify the number of bubbles generated by a leading edge cavity, they propose a two-step procedure. The flow rate of vapor shed by a cavity is first estimated from ventilation tests, assuming that the rate of air necessary to sustain an artificial cavity is the same as the vaporization rate for a natural cavity of equal length. Then, using measurements of bubble population in the wake of a sheet cavity [5] and assuming that the vaporization rate in the cavity is equal to the flow rate of vapor bubbles shed by it, they could get an estimate of the number and size of small scale vapor structures, which are all potential sources of erosion. In spite of such investigations, the detailed mechanism producing small scale structures from a macroscopic cavitation is not yet entirely understood, and it remains very difficult to predict the production rate of small scale vapor structures responsible for cavitation erosion together with their typical size and real nature, bubbles or vortices.

The damage potential of each of these individual structures is also very difficult to predict. Many studies have been conducted on single bubbles in order to investigate the detailed mechanisms of impulsive pressure generation by bubble collapse and associated damage (see, e.g., Refs. 6-8). Bubble dynamics near solid boundaries involves complex phenomena such as the formation of jets, counterjets, ring vortices, etc., whose influence on the cavitation erosion process is not always fully recognized. Several parameters are, however, known to have a major influence on the erosive potential, such as the distance of the bubble to the wall, its maximum size prior to collapse, the adverse pressure gradient to which the bubble is subjected, and which causes its collapse. Collective effects may also affect the violence of the collapse and the associated erosive potential. Reisman et al. [9] showed that the collapse of a cloud of bubbles may enhance the erosive potential of individual bubbles because of the focusing effect of shock waves near the cloud center. In spite many years of fundamental research on this topic, the quantification of the erosive potential of collapsing vapor structures remains a challenge in real cavitating flows.

Energy considerations are often used to approach this problem 
from a global viewpoint in order to predict cavitation damage, disregarding local detailed collapse mechanisms. This type of approach is also the one that is adopted in the present paper. From the classical Rayleigh analysis of the dynamics of a spherical cavity in an inviscid incompressible liquid at rest at infinity, it is well known that the energy discharged as kinetic energy in the fluid by a cavity of volume $V$ collapsing under a pressure difference $\Delta p$ (relative to the pressure inside the bubble) is given by the work of the pressure forces, i.e., $V \times \Delta p$ [10]. According to Stinebring et al. [11], this total cavitation bubble collapse energy is the sum of the energy absorbed by the material, the elastic energy due to the recovery of the surface after the collapse, which produces an acoustic wave propagating through the fluid, and the energy remaining in the bubble after the initial collapse, i.e., the energy of the rebunding bubble(s). The residual plastic energy remaining in material after impact is generally assumed to be proportional to the volume of the resulting pit $[10,12]$. Hammitt [13] introduced the cavitation erosion efficiency, i.e., the ratio between this residual plastic energy and the energy actually applied to the surface by shock waves and microjet impacts. He suggests to estimate the latter from pulse height spectra measured in the collapse region using piezoelectric microtransducers. On the basis of linear acoustic theory, Hammitt [13] proposed to estimate the energy emitted by cavitation bubble collapse from the quantity $p^{2} \times \Delta t /(\rho c)$ calculated using the measured amplitude $p$ of each detected pressure pulse, its duration $\Delta t$, and the acoustic impedance $\rho c$ of the liquid. By considering all pressure pulses detected by the transducer during a given lapse of time and computing the summation of individual collapse energies as defined above, a total energy can be introduced, which is expected to represent the cumulative impact energy of all the vapor structures that have collapsed on the sensitive surface of the transducer during the measuring time. Several investigations concluded on a linear relationship between measured erosion and the total impact energy deduced from pulse height spectra analysis [13-16]. This relation depends on the material but proves to be independent of test conditions including Venturi and vibratory erosion tests. This technique appears then as a valuable method to quantify the erosive potential of a cavitating flow.

Another way to measure the flow aggressiveness is to use pitting tests as proposed 50 years ago by Knapp $[17,18]$. This is the technique that is used in the present work for reasons developed in Sec. 3.2. Since this pioneering work, techniques of analysis of pitted surfaces have been improved [12,19], and the detailed shape of erosion pits is now available. Information on pit size and pit depth allow an improved quantification of the erosive potential in comparison with the only pit density used originally. Using pitting tests as a basis for the determination of flow aggressiveness implies that the material itself is considered as a special transducer whose response to bubble collapses is precisely made of surface pits. A quantitative approach requires us to model the material behavior in order to convert data on pit geometry into data on hydrodynamic loads. This is one of the objectives of the present work.

Another objective is to predict long term damage using the same model of material response. The evolution of cavitation erosion with the exposure time shows different periods. Three main stages are generally distinguished (see, e.g., Refs. 13 and 20). At the very beginning, the erosion damage is made of isolated pits, which progressively overlap as the exposure time increases. Usually, negligible mass loss occurs during this incubation period. Then, mass loss actually begins, and an acceleration period is observed, with a damage rate gradually increasing up to a maximum. A steady-state period with a constant erosion rate is then expected as long as the flow pattern remains unchanged. Secondary effects may affect this schematic behavior, and more complicated evolutions are found in the literature depending, in particular, on the test facility [21]. For example, the steady-state period may be almost inexistent, or the maximum may be followed by a deceleration or even oscillations of the erosion rate [22]. These effects are generally due to an interaction between the cavitating flow and the walls via, for instance, changes in roughness or wall shape induced by the wear itself. They are ignored in the present paper, which focuses on the three basic stages described above. In addition, all erosion tests were started from a highly polished surface, and the effect of the initial surface roughness, which may substantially affect the incubation period [23], was not considered. Corrosion effects were also ignored, and the deterioration of the surface is supposed to result only from the mechanical attack of collapsing bubbles.

This paper is based on an analysis proposed in 1987 by Karimi and Leo [24] and adapted in 2002 by Berchiche et al. [25]. The method is applicable to ductile materials (such as stainless steel $316 \mathrm{~L}$ considered here), which undergo work hardening when exposed to cavitation. Work hardening is characterized by a change in microhardness, dislocation density, and strain with depth below the worn surface [26]. In this paper, it is represented by the shape of the microhardness profile in cross sections of eroded specimens and, in particular, by the thickness of the hardened layer. The starting point of the prediction procedure is made of pitting tests from which the erosive potential of the cavitating flow is characterized in terms of impact loads, as mentioned previously. Berchiche et al. [25] characterized the erosive potential of the cavitating flow by a distribution in size and amplitude of impact loads. They reproduced it numerically a large number of times and could compute mass loss as a function of the exposure time. Because of the wide spectrum of pits, they were obliged to use a numerical procedure that gives realistic evolutions in time and space of the damage but makes difficult a phenomenological analysis. In order to point out the basic parameters of the model and their influence mainly on the incubation time and the steady-state erosion rate, the approach has been reformulated in a simplified way in this paper. The simplification consists in representing the whole spectrum by a limited number of integral parameters. Analytical relationships can then be derived, from which it is much easier to draw general trends and, in particular, to point out the relevant length scale and time scale of the erosion phenomenon for ductile materials.

\section{Phenomenological Analysis}

2.1 Erosive Potential. The real cavitating flow comprises a large variety of vapor structures. When collapsing, each structure is supposed to generate a load on the wall. A large variety of impact loads of various sizes and amplitudes are then generated. The simplification that is made here consists in characterizing the whole impact load spectrum by a mean value $\bar{\sigma}$ of the amplitude and a mean value $\bar{S}$ of the impacted area. A third major parameter with respect to flow aggressiveness is the number $N$ per unit time and unit surface area of these impacts. In summary, the erosive potential of the cavitating flow is characterized here by a set of three parameters, $\bar{\sigma}, \bar{S}$, and $N$. The averaging procedure to represent a complex spectrum by a unique set of values $(\bar{\sigma}, \bar{S}, N)$ is presented in more detail in Sec. 4.1. The general idea is to preserve the total energy impacting the wall.

The present model of an individual impact is the simplest one that can be imagined since the radial distribution of stress is considered as uniform: stress is equal to $\bar{\sigma}$ over the surface $\bar{S}$ and zero outside. Moreover, no indication is given on the time evolution of the applied stress. This means, in particular, that the strain rate, which is known to be very high in cavitating flows, is ignored at this step. The introduction of strain rate in the present analysis would require further developments.

From a purely dimensional viewpoint, a characteristic time can be built on the basis of two of these parameters. It is defined by $\tau=1 / N \bar{S}$. From a physical viewpoint, this time can be interpreted as the covering time. i.e., the time necessary for the surface to be 
fully covered exactly once by the cavitation impacts. This characteristic time plays a fundamental role in the erosion process, as will be shown later.

2.2 Material Thresholds. To estimate damage caused to the wall, it is necessary to compare the amplitude of impact loads to specific material characteristics used as thresholds. In the present paper and following the initial analysis of Karimi and Leo [24], we systematically refer to tensile tests to define these thresholds using the ordinary yield strength $\sigma_{Y}$ and ultimate tensile strength $\sigma_{U}$. More appropriate material tests should probably be used to better account for the very specific type of loading due to bubble collapse. One limitation among others is that ordinary tensile tests are conducted at a strain rate several orders of magnitude smaller than that of cavitation. However, within the same simplified approach, the high strain rate encountered in cavitation erosion can be taken into account, to some extent, by artificially increasing $\sigma_{Y}$ and $\sigma_{U}$ with respect to the values given by a classical tensile test at much smaller strain rate.

In the present paper, a Ludwik-type consolidation relationship between stress $\sigma$ and strain $\varepsilon$ is chosen,

$$
\sigma=\sigma_{Y}+K \varepsilon^{n}
$$

Let us observe that although Eq. (1) is, strictly speaking, valid only up to the ultimate strength, it will be extrapolated beyond $\sigma_{U}$. This is the basis for the computation of mass loss rate.

Throughout this paper, we shall take the example of stainless steel $316 \mathrm{~L}$, which has been used for the experimental part of this work. Typical values for SS $316 \mathrm{~L}$ are [25]

$$
\begin{gathered}
\sigma_{Y}=400 \mathrm{MPa} \\
\sigma_{U}=1020 \mathrm{MPa} \\
K=900 \mathrm{MPa} \\
n=0.5
\end{gathered}
$$

The erosion damage depends primarily on the mean amplitude $\bar{\sigma}$ of the impact loads relative to the two previous thresholds. Three cases can happen.

(i) If $\bar{\sigma}<\sigma_{Y}$, the impacts are supposed to cause no damage at all, whatever their size and rate may be. The elastic behavior dominates.

(ii) If $\bar{\sigma}$ lies between $\sigma_{Y}$ and $\sigma_{U}$, successive impacts cause first the progressive hardening of the initially virgin material without any mass loss and then its rupture, and the penetration of the damage after the work-hardening process is completed. This fatigue-type mechanism is the way the incubation time is accounted for in the present model.

(iii) Finally, if $\bar{\sigma}>\sigma_{U}$, mass loss appears from the very beginning of exposure to cavitation.

2.3 Work Hardening. For a virgin material, strain is zero everywhere inside the material. As it is exposed to bubble collapses, the successive hydrodynamic impacts lead to a progressive superficial hardening. The strain profile inside an eroded sample can be determined from microhardness measurements on a cross section [25]. A classical representation of the strain profile inside the material is

$$
\varepsilon(x)=\varepsilon_{0}\left[1-\frac{x}{\ell}\right]^{\theta}
$$

where $\varepsilon_{0}$ is the strain on the surface, $x$ is the distance below the surface, $\ell$ is the thickness of the hardened layers, and $\theta$ is a shape factor for the strain profile.

The thickness $\ell$ of the hardened layer progressively increases with exposure time and reaches a maximum (denoted as $L$ ) when the work-hardening process is completed. The surface strain is

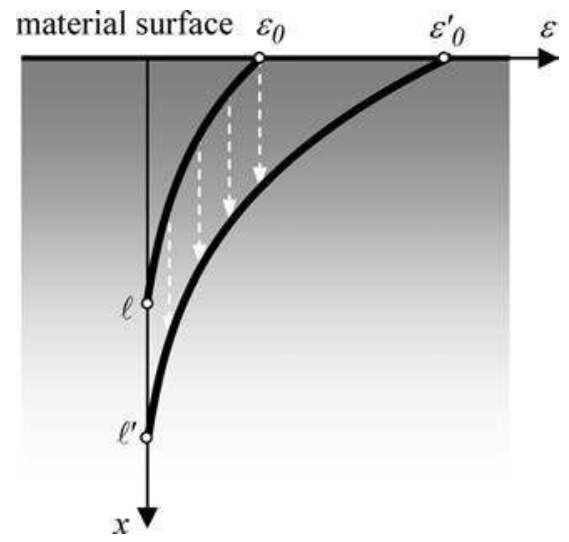

Fig. 1 Work-hardening progress

then $\varepsilon_{U}$ corresponding to $\sigma_{U}$ : the material is then ready to rupture at its surface. Typical values of $L$ and $\theta$ for stainless steel $316 \mathrm{~L}$ are [25]

$$
\begin{gathered}
L=200 \mu \mathrm{m} \\
\theta=5
\end{gathered}
$$

To predict the evolution of $\ell$ with $\varepsilon_{0}$, it is observed that the strain profile can be considered as simply translated inside the material as work hardening proceeds. This is a property of the power law chosen to describe the strain profile. In other words, if $\varepsilon_{0}$ increases up to $\varepsilon^{\prime}{ }_{0}$ whereas $\ell$ increases up to $\ell^{\prime}$ (Fig. 1), the new strain profile is

$$
\varepsilon(x)=\varepsilon^{\prime}{ }_{0}\left[1-\frac{x}{\ell^{\prime}}\right]^{\theta}
$$

and we have $\varepsilon(x)=\varepsilon_{0}$ for $x=\ell^{\prime}-\ell$, i.e.,

$$
\frac{\varepsilon_{0}}{\varepsilon^{\prime}{ }_{0}}=\left[\frac{\ell}{\ell^{\prime}}\right]^{\theta}
$$

Equation (6) shows that the thickness $\ell$ of the hardened layer increases as $\varepsilon^{1 / \theta}$. In particular, $\ell$ is connected to the maximum value $L$ by

$$
\frac{\varepsilon_{0}}{\varepsilon_{U}}=\left[\frac{\ell}{L}\right]^{\theta}
$$

2.4 Single Impact. As already mentioned in Sec. 1, the present approach is based on energy considerations. A key concept is the energy absorbed by the material when submitted to a single hydrodynamic impact $(\bar{\sigma}, \bar{S})$. This energy is assumed to be independent of the degree of hardening of the material and is evaluated on the basis of a virgin material. It is also assumed to be independent of surface roughness.

Let us denote by $\varepsilon_{1}$ the strain on the material surface resulting from an impact of amplitude $\bar{\sigma}$ on the virgin material. There is a unique correspondence between $\bar{\sigma}$ and $\varepsilon_{1}$ via the material stress/ strain relationship (1). The $\varepsilon_{1}$-variable can then be used instead of $\bar{\sigma}$. In case $\bar{\sigma}>\sigma_{U}$, the approach remains valid provided the stress/ strain relationship is extrapolated beyond $\sigma_{U}$.

After a single impact, the material surface behaves as indicated schematically in Fig. 2(a). The surface strain is $\varepsilon_{1}$ corresponding to impact load $\bar{\sigma}$. The energy absorbed per unit volume by the most superficial layer is the area below the stress/strain curve. As for internal layers, strain $\varepsilon(x)$ decreases with distance $x$ from the surface according to Eq. (3), and the energy absorbed (which is still the area below the stress/strain curve) is smaller for inner layers than for the most superficial ones (Fig. 2(b)).

The total energy absorbed by the material can be computed as 

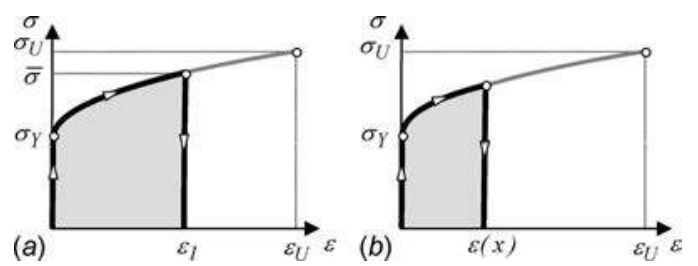

Fig. 2 Behavior of a virgin material after being loaded by a hydrodynamic impact of amplitude $\bar{\sigma}$. (a) Case of the superficial layer. (b) Case of a layer at depth $x$.

$$
W\left(\varepsilon_{1}\right)=\int_{x=0}^{\ell}\left[\int_{\varepsilon=0}^{\varepsilon(x)} \sigma d \varepsilon\right] \bar{S} d x
$$

It is given by

$$
W\left(\varepsilon_{1}\right)=\varepsilon_{1} \bar{S} L\left(\frac{\varepsilon_{1}}{\varepsilon_{U}}\right)^{1 / \theta} \frac{\sigma_{Y}+\beta K \varepsilon_{1}^{n}}{1+\theta}
$$

with

$$
\beta=\frac{1+\theta}{(1+n)(1+\theta+n \theta)}
$$

$L$ is the maximum thickness of the hardened layers when work hardening is complete, whereas the quantity

$$
L\left(\frac{\varepsilon_{1}}{\varepsilon_{U}}\right)^{1 / \theta}
$$

is the thickness of the hardened layers for a partial hardening due to the only considered impact $\bar{\sigma}$ (see Eq. (7)).

2.5 Incubation Period. As exposure time increases, the material is hardened and surface strain progressively increases. After the first covering of the initially virgin material (i.e., after time $\tau$ ), surface strain is uniformly equal to $\varepsilon_{1}$. After the second covering (time $2 \tau$ ), surface strain is increased from $\varepsilon_{1}$ up to a value of $\varepsilon_{2}$, which is deduced from the conservation of energy. Since there has been two coverings, the flow has discharged into the material the energy $2 W\left(\varepsilon_{1}\right)$. Moreover, if surface strain is $\varepsilon_{2}$, the total energy absorbed by the material is actually $W\left(\varepsilon_{2}\right)$. The energy balance writes $W\left(\varepsilon_{2}\right)=2 W\left(\varepsilon_{1}\right)$. This equation allows the determination of the $\varepsilon_{2}$-value. The work-hardening process is continued between the second covering (instant $2 \tau$ ) and the third one (instant $3 \tau$ ). Surface strain increases up to a $\varepsilon_{3}$-value given by $W\left(\varepsilon_{3}\right)$ $=3 W\left(\varepsilon_{1}\right)$, and so on (see Fig. 3).

No mass loss is expected until surface strain reaches the ultimate strain $\varepsilon_{U}$ corresponding to the ultimate tensile strength $\sigma_{U}$. The number of coverings necessary to reach this critical point is $n=W\left(\varepsilon_{U}\right) / W\left(\varepsilon_{1}\right)$, and the corresponding time is $n \tau$. This time is

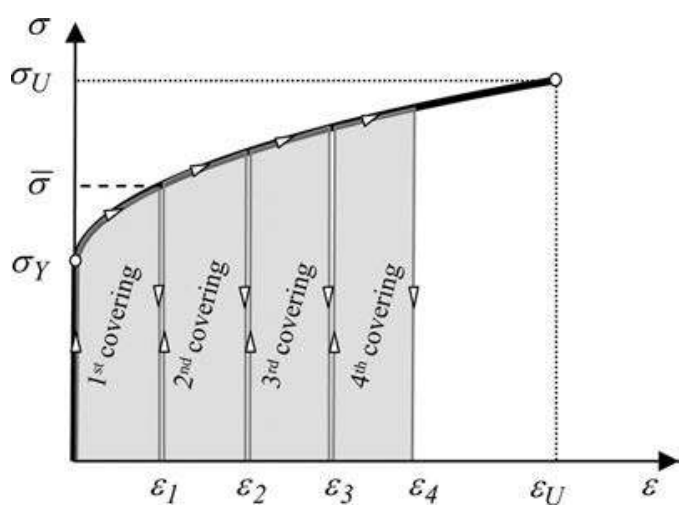

Fig. 3 Material response during the incubation period

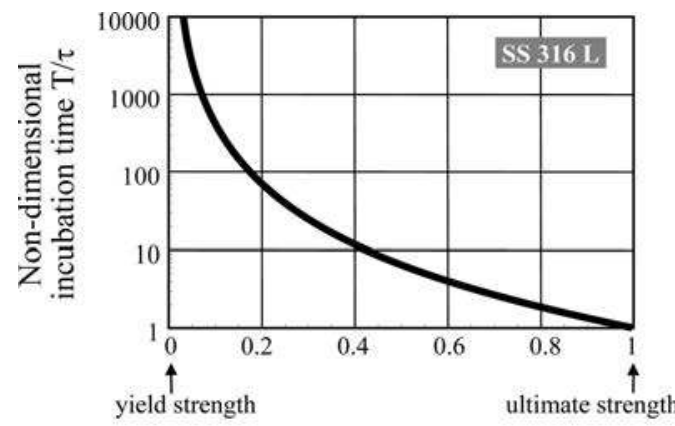

Non-dimensional amplitude of impact loads

Fig. 4 Typical example of the variation of the incubation period with the amplitude of impact loads $\bar{\sigma}$. Case of stainless steel $316 \mathrm{~L}$. The values of the material constants are given in Eqs. (2) and (4). The abscissa represents the nondimensional amplitude of impact loads defined by $\left(\bar{\sigma}-\sigma_{Y}\right) /\left(\sigma_{U}-\sigma_{Y}\right)$, whereas the ordinate is the incubation time $T$ made nondimensional using the covering time $\tau$.

precisely the incubation period $T$ beyond which mass loss is expected. Using Eq. (9), together with the stress/strain relationship (1), the incubation time is given in a nondimensional form by

$$
\frac{T}{\tau}=\left[\frac{\sigma_{U}-\sigma_{Y}}{\bar{\sigma}-\sigma_{Y}}\right]^{(1+\theta) / n \theta} \frac{\sigma_{Y}+\beta\left(\sigma_{U}-\sigma_{Y}\right)}{\sigma_{Y}+\beta\left(\bar{\sigma}-\sigma_{Y}\right)}
$$

Equation (12) accounts for both the influence of the erosive potential of the cavitating flow and that of the material properties. The incubation period depends on the material properties $\sigma_{Y}, \sigma_{U}$, $n$, and $\theta$ (but not on the thickness $L$ of the hardened layers) and on the flow aggressiveness $\bar{\sigma}, \bar{S}$, and $N$. Concerning the last two parameters, the incubation period appears to be simply proportional to the covering time $\tau=1 / N \bar{S}$. The dependence with respect to the amplitude of impact loads $\bar{\sigma}$ is more complex. When $\bar{\sigma}$ approaches $\sigma_{Y}$, the incubation time tends to infinity. This is consistent with the assumption that no damage will occur if the amplitude of impacts is smaller than the yield strength. On the other hand, for $\bar{\sigma}=\sigma_{U}$, one has $T=\tau$. This means that mass loss is expected to happen after the material surface has been covered only once. This is quite understandable from a physical viewpoint. Beyond $\sigma_{U}$, the incubation time takes no sense since erosion occurs from the very beginning of exposure. Between $\sigma_{Y}$ and $\sigma_{U}$, the incubation period progressively decreases as flow aggressiveness (here $\bar{\sigma}$ ) increases. As an example, the evolution of the incubation period with the mean amplitude of impact loads is presented in Fig. 4 for stainless steel $316 \mathrm{~L}$ using the numerical values given in Eqs. (2) and (4). In case the mean amplitude of impact loads is only a little higher than the yield strength, i.e., for flows of relatively small erosive potential, the number of coverings necessary before mass loss happens may be quite large, and the incubation time may be several orders of magnitude larger than the covering time.

2.6 Steady-State Mass Loss Rate. After the incubation period, surface strain is maximum and equal to $\varepsilon_{U}$. Any further impact will cause material removal. After a new complete covering of the material surface, strain at surface reaches a value of $\varepsilon^{\prime}$ greater than $\varepsilon_{U}$ (see Fig. 5). This $\varepsilon^{\prime}$-value is extrapolated beyond the ultimate tensile strength on the stress/strain relationship. It can be considered as virtual in so far as all the layers of the material with a strain greater than $\varepsilon_{U}$ are indeed assumed to be ruptured and eroded. This $\varepsilon^{\prime}$-value is computed, as previously, from an energy balance, which is written as

$$
W\left(\varepsilon^{\prime}\right)=W\left(\varepsilon_{U}\right)+W\left(\varepsilon_{1}\right)
$$




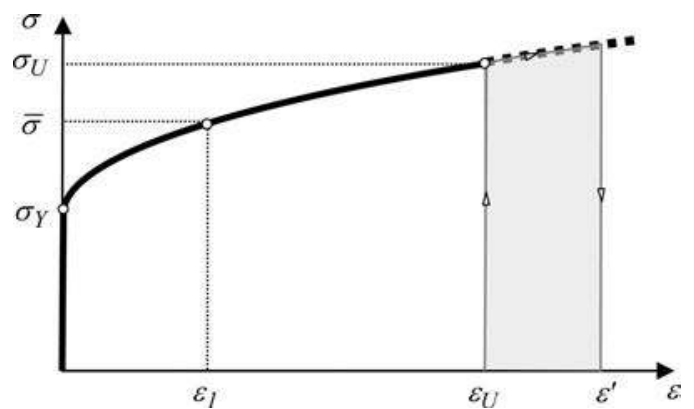

Fig. 5 Material behavior after the incubation period during steady-state erosion

Once the new surface strain $\varepsilon^{\prime}$ is known, the new strain profile inside the material can be computed. Both profiles, just after the incubation period and after an additional covering, are compared in Fig. 6. They are simply translated with a length $\Delta L$ given by

$$
\frac{\Delta L}{L}=\left[\frac{\varepsilon^{\prime}}{\varepsilon_{U}}\right]^{1 / \theta}-1
$$

as explained in Sec. 2.3. The previous equation is a direct consequence of Eq. (7).

Mass loss is computed by assuming that the material cannot sustain a strain greater than the ultimate strain $\varepsilon_{U}$ so that the thickness $\Delta L$ is actually eroded and removed during the interval of time $\tau$. The mean depth of penetration rate (MDPR) is then

$$
\operatorname{MDPR}=\frac{\Delta L}{\tau}=\frac{L}{\tau}\left[\left(\frac{\varepsilon^{\prime}}{\varepsilon_{U}}\right)^{1 / \theta}-1\right]
$$

A major outcome of this equation is that the steady-state erosion rate MDPR is proportional to $L / \tau$. In other words, from a dimensional viewpoint, it appears that the characteristic length, which is pertinent for the computation of MDPR is the thickness $L$ of the hardened layers (which is a feature of the material), whereas the characteristic time to be considered is the covering time $\tau$ (which is a feature of the fluid flow). The term in brackets is a multiplicative factor, which depends primarily on flow aggressiveness. It is estimated below in the case of stainless steel $316 \mathrm{~L}$.

A practical way to compute MDPR is as follows. From the mean value of the impact load $\bar{\sigma}$, strain $\varepsilon_{1}$ is computed using the stress/strain relationship, and the corresponding energy $W\left(\varepsilon_{1}\right)$ is computed using Eq. (9). The energy conservation equation (Eq. (13)) is then solved in order to determine $\varepsilon^{\prime}$. Since this equation cannot be solved analytically, we cannot get a fully analytical equation for MDPR. The mean depth of penetration rate can then

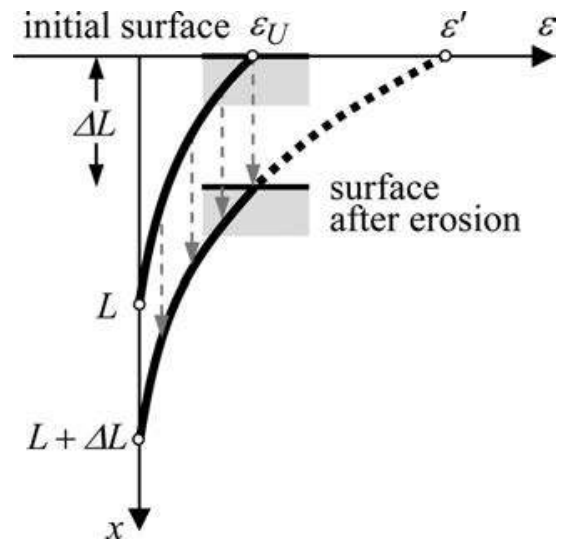

Fig. 6 Principle of the computation of mass loss

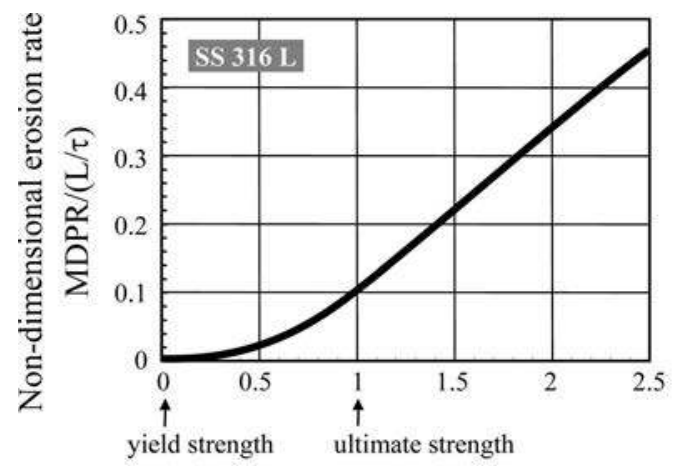

Non-dimensional amplitude of impact loads

Fig. 7 Typical example of the variation in the MDPR with the mean amplitude of impact loads. Case of stainless steel $316 \mathrm{~L}$ (see Eqs. (2) and (4) for values of constants). MDPR is made nondimensional using the characteristic erosion rate $L / \tau$, whereas the nondimensional amplitude of impact loads is defined as in Fig. 4.

be computed using Eq. (15).

A typical evolution of the erosion rate with flow aggressiveness is presented in Fig. 7, still in the case of stainless steel $316 \mathrm{~L}$. In the limit case $\bar{\sigma}=\sigma_{Y}$, one has $W\left(\varepsilon_{1}\right)=0, \varepsilon^{\prime}=\varepsilon_{U}$, and then $\mathrm{MDPR}=0$, as expected. Figure 7 shows that MDPR increases first slowly with $\bar{\sigma}$ around the yield strength and tends to be more or less linear with $\bar{\sigma}$ beyond the ultimate strength. For $\bar{\sigma}<\sigma_{U}$, damage is due to fatigue associated with the accumulation of energy in the material. The present approach remains valid for severe cavitation erosion $\left(\bar{\sigma}>\sigma_{U}\right)$.

\section{Experiments}

3.1 Experimental Facility. To support the previous phenomenological analysis, erosion tests were conducted in a cavitation tunnel (Fig. 8). The facility is designed for a maximum operating pressure of 40 bars. This relatively high pressure provides high velocities and, consequently, high erosive potential for the cavitating flow. This is an essential condition to obtain significant mass loss within reasonable exposure times and to make possible the investigation of the advanced stages of erosion. The pump is a centrifugal pump driven by an electric motor of $80 \mathrm{~kW}$ in power. The maximum flow rate is $11 \mathrm{l} / \mathrm{s}$. The facility includes a downstream tank of about $1 \mathrm{~m}^{3}$. Pressurization is achieved by means of a bottle connected to the tank by a pipe of small diameter in order to limit the diffusion of nitrogen used for pressurization. Liquid is tap water without any special control of dissolved gas. Water temperature is kept constant by means of a heat exchanger. It is a countercurrent exchanger made of 85 tubes of $11 \mathrm{~mm}$ in diameter with a nominal power of $80 \mathrm{~kW}$. Tests are conducted at ambient temperature. The rise in temperature after a run of $5 \mathrm{~h}$ is typically of the order of $0.5^{\circ} \mathrm{C}$. The tunnel is equipped with several transducers to determine the operating conditions: an electromagnetic flowmeter, a pressure transducer to measure the absolute pressure upstream of the test section $p_{u}$, a differential pressure transducer to measure the pressure drop through the test section, and a temperature probe.

The test section is about $6 \mathrm{~m}$ above the pump in order to avoid any cavitation of the pump. It is axisymmetric and made of a nozzle of $16 \mathrm{~mm}$ in diameter followed by a radial divergent of 2.5 $\mathrm{mm}$ in thickness (Fig. 9). Under cavitating conditions, a cavity is attached to the nozzle exit whose length can be adjusted by changing the cavitation number, which is obtained in practice by changing the downstream pressure $p_{d}$. Cavitation number is defined by 


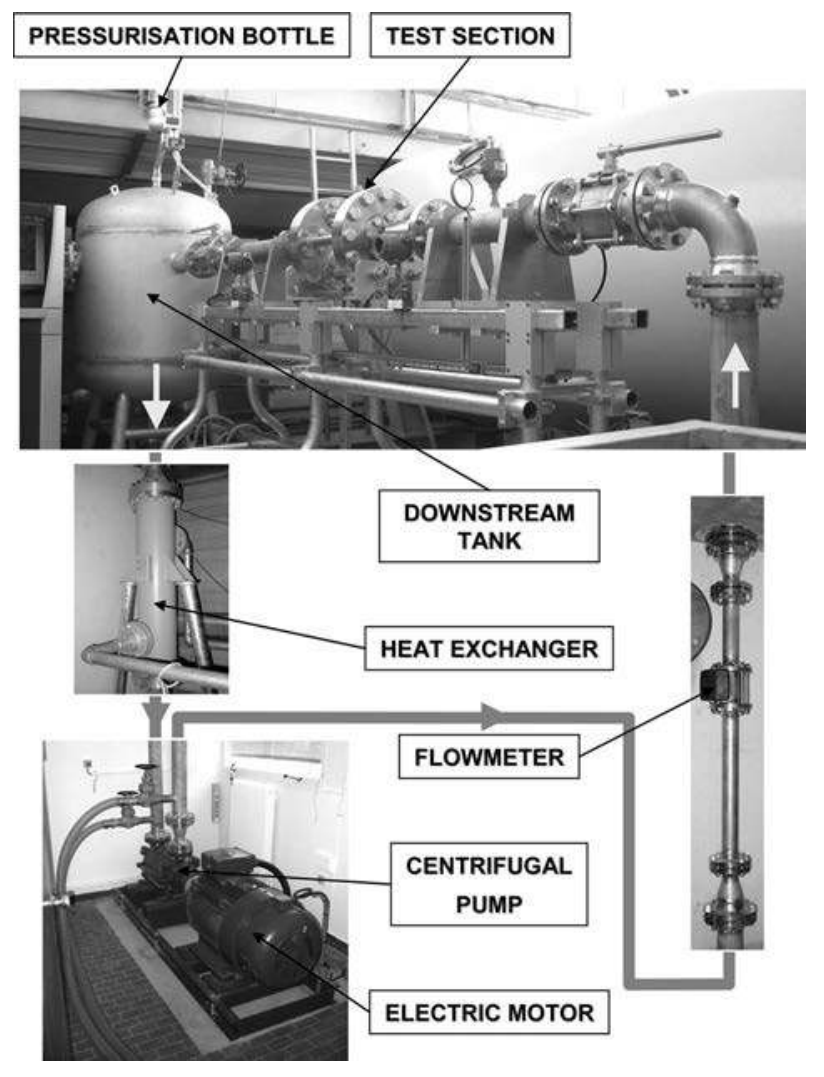

Fig. 8 View of the cavitation erosion tunnel

$$
\sigma=\frac{p_{d}-p_{v}}{p_{u}-p_{d}}
$$

The $\sigma$-value is chosen to be equal to 0.9 , which leads to a cavity of about $25 \mathrm{~mm}$ in mean length. This ensures that the zone of maximum erosion, which corresponds to cavity closure lies in an appropriate region of the target for the subsequent analysis. The tests presented in this paper were conducted at a flow rate of 6.25 1/s. The upstream pressure is 21.3 bars, and the pressure drop through the test section given by

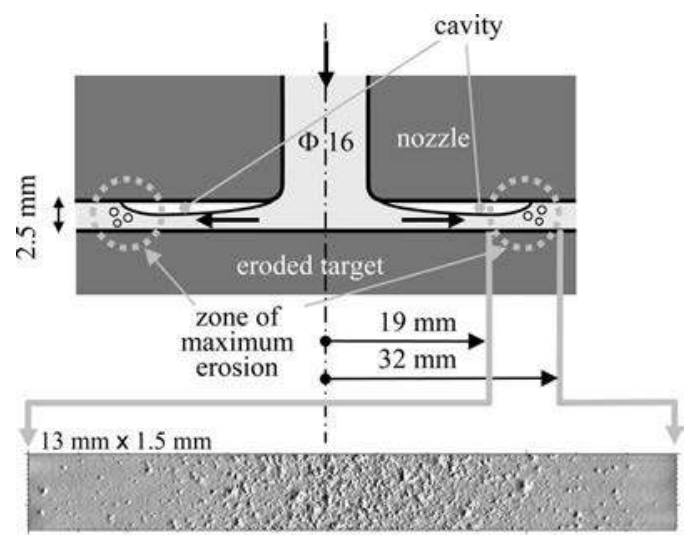

$13 \mathrm{~mm} \times 1.5 \mathrm{~mm} \times 3 \mu \mathrm{m}$

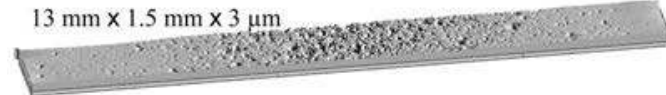

Fig. 9 Schematic view of the test section together with an eroded target. The size of the eroded surface shown is 13 $\times 1.5 \mathrm{~mm}^{2}$. The operating conditions are $V_{c}=65.3 \mathrm{~m} / \mathrm{s}, p_{u}$ $=21.3$ bars, $p_{d}=10.1$ bars, and $\sigma=0.9$. Test duration: $30 \mathrm{~min}$.

$$
p_{u}-p_{d} \cong \frac{1}{1+\sigma} p_{u}
$$

is 11.2 bars at $\sigma=0.9$. The mean velocity in the $16 \mathrm{~mm}$ nozzle is $31 \mathrm{~m} / \mathrm{s}$. The reference velocity on the cavity where pressure is assumed to be equal to the vapor pressure $p_{v}$ can be estimated from the Bernoulli equation in which the vapor pressure is neglected compared with the upstream pressure,

$$
V_{c} \cong \sqrt{\frac{2 p_{u}}{\rho}}
$$

For present tests, the reference velocity on the cavity is $V_{c}$ $=65.3 \mathrm{~m} / \mathrm{s}$.

The target to be eroded faces the nozzle exit. Erosion appears in the form of a ring centered on cavity closure where erosive potential is maximum (see Fig. 15). Both sides of the channel are expected to be eroded, but the nozzle is made of a highly resistant material so that no significant erosion is observed on the nozzle itself. Erosion tests are conducted on stainless steel $316 \mathrm{~L}$, free of any corrosion effect in water. Prior to exposure to cavitation, a metallurgical polishing of the specimen surface is carried out with successive diamond pastes down to $0.25 \mu \mathrm{m}$, which ensures a mirror-type polish. The effect of roughness on cavitation erosion damage is not considered in the present investigation.

3.2 Pitting Tests. Pitting tests are used here to characterize the erosive potential of the cavitating flow. The idea of using the material itself as a kind of transducer to reveal the flow aggressiveness is classical [10]. It consists in considering that each indentation is the signature of the collapse of a bubble. In a first approach, it can reasonably be assumed that pit depth is representative of maximum load and pit size of the extent of the loaded area. This is the assumption made in Sec. 4.1 below.

As mentioned in Sec. 1, an alternative for characterizing flow aggressiveness is to use pressure transducers. Li et al. [27] showed that the temporal pressure fluctuations given by a transducer flush mounted in the cavitating region is made of several components. In addition to basic flow noise and low-frequency fluctuations associated with the global behavior of the two-phase region, they observed high-frequency pulses due to cavitation bubble collapse. Several investigators (see, e.g., Refs. 15, 28, and 29) have shown that a good correlation exists between erosion damage and the number of pulses above a suitable threshold. However, the quantitative evaluation of pressure load due to bubble collapse by pressure transducers raises a few basic difficulties. The very high characteristic frequency of cavitation pulses requires transducers of small rise time in order to follow reliably the fast pressure rise. Very significant progress has been made in this field by the development of special transducers $[15,30]$. But a major difficulty remains concerning the size of the sensitive surface, which, although miniaturized, is much bigger than that of indentations. Even though realistic estimates can be obtained by dividing the measured load in terms of force by the area of the indentation [30], pressure transducers cannot directly supply pressure load. This problem leads us to prefer pitting tests to evaluate flow aggressiveness. Nevertheless, the estimate of pressure load from indentation characteristics is not straightforward as well, and a special procedure (see Sec. 4.1) based on a modeling of the material response is needed.

Figure 10 presents a typical view of a pitted surface (see also top of Fig. 22). A pitting test can be considered as acceptable if the degree of pit overlapping is low enough. It is clear that the response of a ductile material is not the same whether the impact falls on a virgin part of its surface or an already pitted area. This is because of the increase in superficial hardness due to pitting. Since the analysis of the pitting test presented in Sec. 4.1 is based on the assumption that each pit falls on a virgin area, it is of major importance to limit overlapping in order to obtain an unbiased estimate of the erosive potential. The degree of overlapping is 


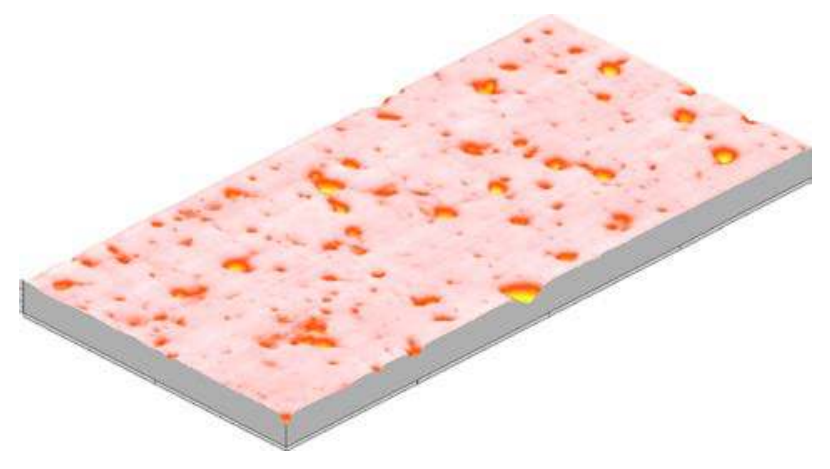

Fig. 10 Typical 3D view of the surface after a pitting test. The size of the volume shown is $2 \mathrm{~mm} \times 4 \mathrm{~mm} \times 2.8 \mu \mathrm{m}$. Same operating conditions as in Fig. 9. Exposure time: 5 min.

controlled by the exposure time, and, statistically speaking, it can be reasonably assumed that it is negligible if the exposure time is small enough. In practice, it has to be compared with the covering time introduced in Sec. 2.1. In the case of Fig. 10, the covering time is estimated to $88 \mathrm{~min}$, whereas the exposure time is only 5 min. It can then be concluded that overlapping is negligible. The photograph in Fig. 10 confirms that, at least qualitatively, overlapping is only occasional. Provided the exposure time is much smaller than the covering time, it can be expected that the analysis of a pitting test to evaluate flow aggressiveness is independent of exposure time. The smaller the exposure time, the smaller the pit number and the less accurate the estimate. Conversely, the larger the exposure time, the larger the degree of overlapping and the less accurate the estimate as well. It is then necessary to find a compromise. A possibility can be to make several pitting tests of rather short duration and to cumulate the data to get a representative sample from a statistical viewpoint, as will be done later. Furthermore, it is essential that the exposure time be sufficiently short so that large portions of the original virgin surface remain easily detectable between indentations. This is important for the analysis technique since the original surface is used as a reference for the determination of pit depth.

Several techniques are available to analyze a pitted surface. Belahadji et al. [19] used an interferometric technique, and Fortes Patella et al. [12] used a laser profilometer. In the present work, a contact-type profilometer has been used with a stylus of tip radius of $2 \mu \mathrm{m}$ and a vertical maximum resolution of $3.2 \mathrm{~nm}$. The surface is scanned along parallel lines distanced $1 \mu \mathrm{m}$ apart with a resolution of $0.5 \mu \mathrm{m}$ between two successive points. For most tests, a surface of $2 \times 4 \mathrm{~mm}^{2}$ was scanned. Treatment of the data starts by subtracting a polynomial obtained by the least mean square technique. After several tests, a polynomial of degree 5 was considered. It proved to satisfactorily remove the mean shape of the surface often due to large scale machining or polishing defects without altering the shape of pits at a much smaller scale. The next step consists in applying a threshold to the surface in order to define the pit border. For all the analyses presented here, the threshold was fixed at $0.5 \mu \mathrm{m}$ below the reference surface corresponding to the original material surface. All pits whose depth is smaller than $0.5 \mu \mathrm{m}$ are then ignored. This threshold is constant and independent of the indentation. Its influence on the subsequent prediction of incubation time and mass loss rate is discussed in Sec. 4.2. The chosen value of $0.5 \mu \mathrm{m}$ proved to give a correct description of the pits (see Fig. 22). Other investigators $[12,19]$ consider a pit dependent threshold often taken as a fraction (typically 10\%) of maximum pit depth. The influence of the analysis technique on the estimate of the erosive potential of the flow and the subsequent estimate of long term damage has not been investigated.

By this technique, each pit is identified, and its main characteristics (surface, volume, maximum depth, and mean diameter) are

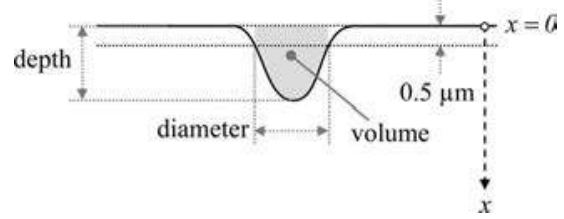

Fig. 11 Sketch showing the definition of pit depth, diameter, and volume

computed. Pit size is defined as the equivalent diameter of the section of the pit by the plane $x=0.5 \mu \mathrm{m}$. Pit depth is counted from the original material surface $x=0$, and volume is defined as shown in Fig. 11.

Figure 12 gives a representation of a sample of almost 800 pits analyzed here in a diagram whose coordinates are pit depth and pit diameter. No correlation is noticeable between pit depth and pit diameter so that both parameters can be considered as independent. In other words, large pits are not necessarily deeper, and no definite geometric similarity is observed on pit profiles.

The distribution of pits as a function of diameter is shown in Fig. 13. The smaller the pits, the higher the pitting rate. As for the contribution of each class of size to the damage, it can be estimated by considering the eroded surface defined here as the total surface of pits belonging to each class of size. The corresponding cumulative and probability density functions (PDFs) are shown in Fig. 14. PDF exhibits a maximum for pit diameters around $100 \mu \mathrm{m}$. Small pits are many but do not contribute significantly to the eroded surface because of their small size. The contribution of large pits is also negligible, but because of their small probability. The existence of a maximum is then quite understandable. It has also been observed by Belahadji et al. [19] for pitting tests in a cavitating Venturi. The conclusion is the same when considering the eroded volume defined as the total volume of pits belonging to each class of size. In particular, the PDF of the eroded volume exhibits a maximum in the same range of size, around $100 \mu \mathrm{m}$, as shown in Fig. 15. For the prediction of incubation time and mass loss rate, it is essential that the technique of analysis of pitting tests focuses on the range of pit diameters, which contribute most to the damage. In the present case, the contribution of pits smaller than $20 \mu \mathrm{m}$ was ignored, whereas the largest observed pit was $220 \mu \mathrm{m}$ in diameter (see Fig. 12).

Note that pitting tests are conducted here on the same material as the one used for mass loss experiments. This obviously simpli-

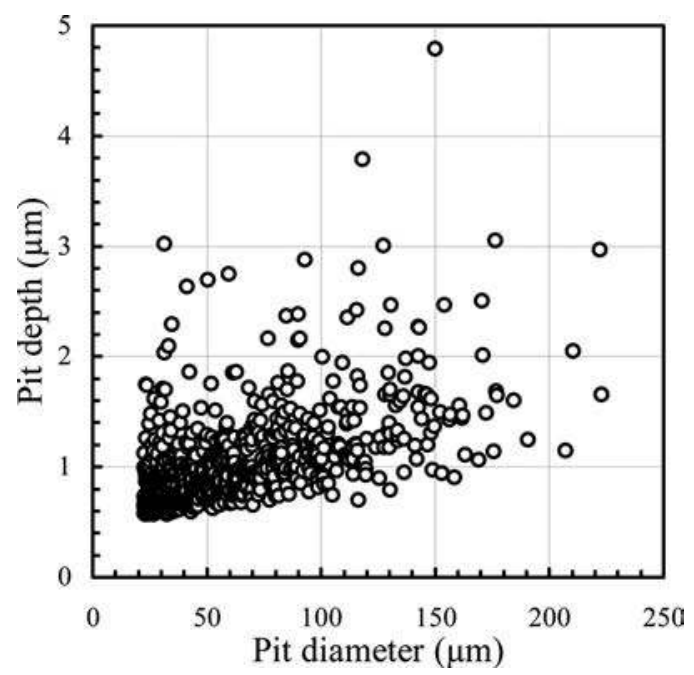

Fig. 12 Pit depth versus pit diameter. Each point represents a pit. The total number of pits is 797 . 


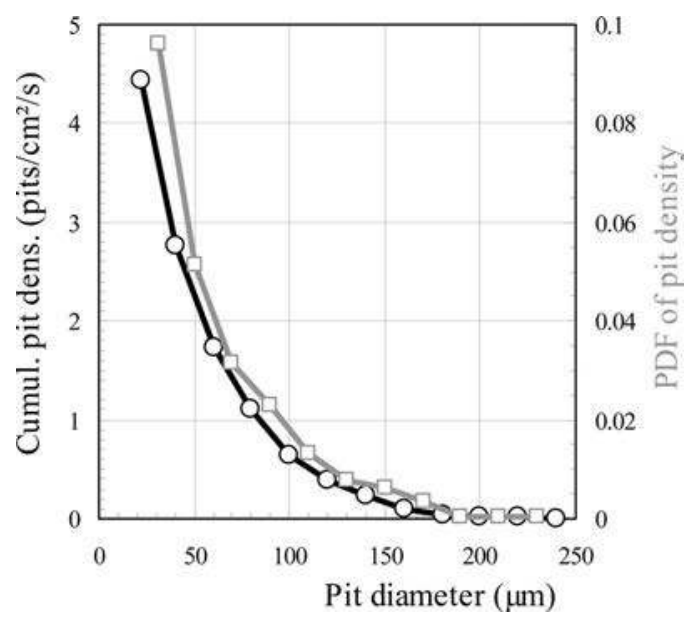

Fig. 13 Cumulative distribution and probability density functions of pit density per unit time and unit surface area. Same operating conditions as in Fig. 9. Total number of pits: 797. Analyzed surface: $59.9 \mathrm{~mm}^{2}$.

fies the approach in comparison with pitting tests conducted on another material, for instance, a softer one as it is often done in order to reduce test duration. The problem of the dependency of the estimated flow aggressiveness on the material used for pitting tests as well as the question of transposition from a material to another are not addressed in the present paper.

3.3 Mass Loss Experiments. A series of mass loss experiments with increasing exposure times has been conducted on a SS $316 \mathrm{~L}$ target for the same operating conditions as the pitting tests presented in Sec. 3.2. Figure 16 presents a view of the material surface after the maximum exposure time of $104 \mathrm{~h}$. Damage is concentrated on a ring corresponding to the closure region of the cavity with almost no damage inside the cavity. On the whole, damage appears axisymmetric enough despite a few undulations in the upper left quarter, which may be due to erosion of the nozzle even though very limited. The measurements were then concentrated on the three other quarters free of defects.

The mass loss test was bracketed by two pitting tests in order to detect any possible alteration in flow aggressiveness during the long duration test. No significant variation was observed between both pitting tests so that it can be considered that flow aggressiveness has remained invariable all along the mass loss test. This

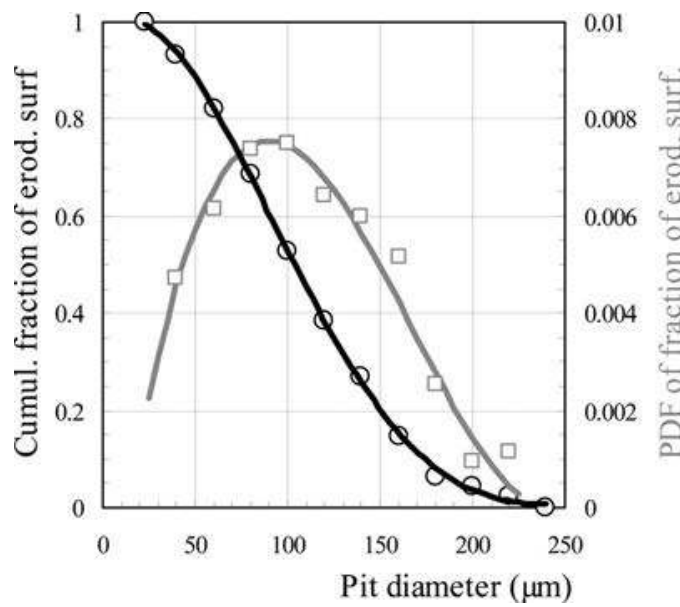

Fig. 14 Cumulative distribution and probability density functions of the fraction of eroded surface. Same conditions as in Fig. 12.

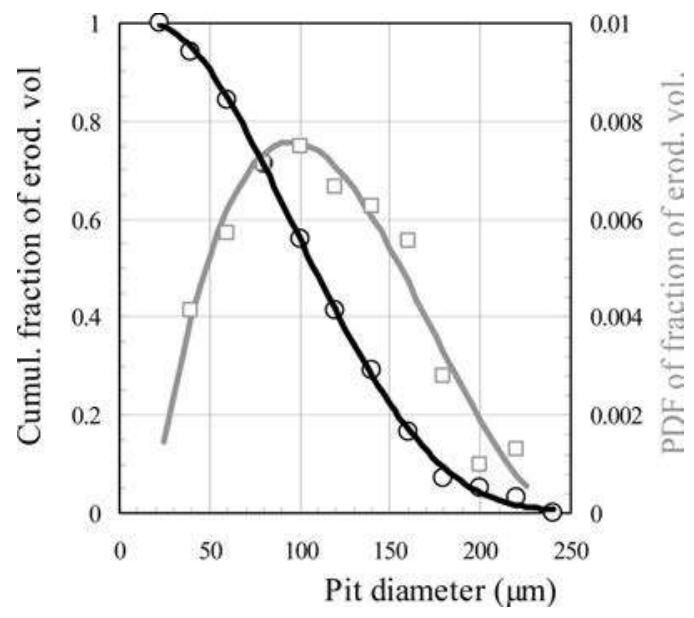

Fig. 15 Cumulative distribution and probability density functions of the fraction of eroded volume. Same conditions as in Fig. 12.

results from (i) a negligible change in wall geometry since the maximum depth of penetration was $70 \mu \mathrm{m}$ after $104 \mathrm{~h}$, which is negligible in comparison with the $2.5 \mathrm{~mm}$ gap (Fig. 9), and (ii) a precise control of the operating conditions, which prevent any shift during operation.

Radial profiles of erosion are presented in Fig. 17 for an increasing exposure time. During about the first $30 \mathrm{~h}$ of exposure to cavitation, there is no measurable penetration of damage, which is essentially characterized by an increase in roughness. After this incubation period, some material is removed and the depth of penetration regularly increases. Raw profiles shown in Fig. 17 reveal the strong roughness of the eroded surface; they need to be smoothed for further analysis. An averaging technique on a $1 \mathrm{~mm}$ wide moving window was systematically applied before estimating the depth of penetration. Let us observe that because of the massive feature and large weight of the sample, mass loss could not be measured with a sufficient accuracy by a weighting technique. Strictly speaking, present estimates are then volume loss

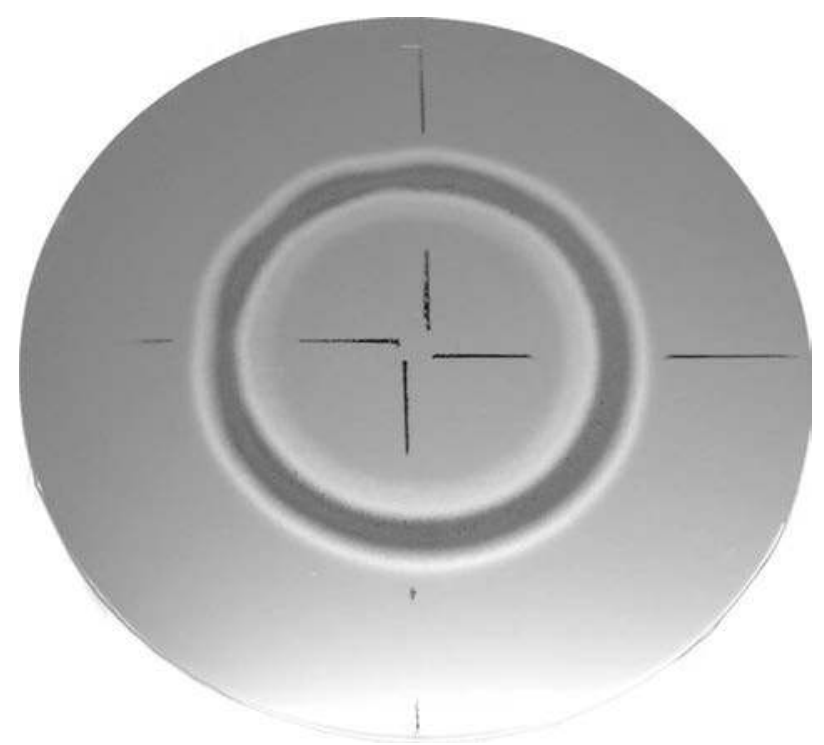

Fig. 16 Photograph of an eroded sample after an exposure time of $104 \mathrm{~h}$. The external diameter of the sample is $100 \mathrm{~mm}$. Operating conditions: $V_{c}=65.3 \mathrm{~m} / \mathrm{s}, \quad p_{u}=21.3$ bars, $p_{d}$ $=10.1$ bars, and $\sigma=0.9$. 


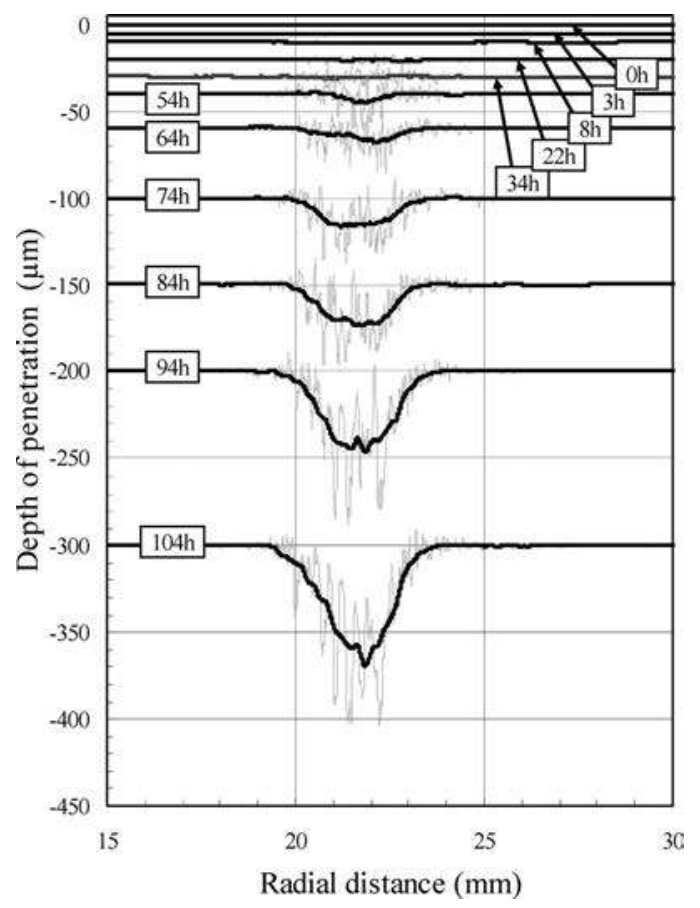

Fig. 17 Influence of the exposure time on radial profiles of erosion for the sample presented in Fig. 19. For each time, two profiles are presented: (i) light gray: raw data with a step of $10 \mu \mathrm{m}$; (ii) thick black: moving average data on 100 points or 1 $\mathrm{mm}$. (The origins of horizontal and vertical scales are arbitrary.)

and not mass loss measurements.

The comparison of three different radial profiles (not shown here) confirms a satisfactory axisymmetry of the erosion pattern, as already concluded from the photograph of Fig. 16. Data on MDPR presented below are relative to the average of three smoothed profiles. Two quantities are considered: (i) the maximum depth of penetration and (ii) a mean depth of penetration defined over a radial distance of $2 \mathrm{~mm}$ centered on the point of maximum erosion. This last procedure presents the advantage of smoothing the data and reducing the dispersion since the damage is defined on a more global basis. Both estimates of depth of penetration are shown in Fig. 18. The smooth one is favored for

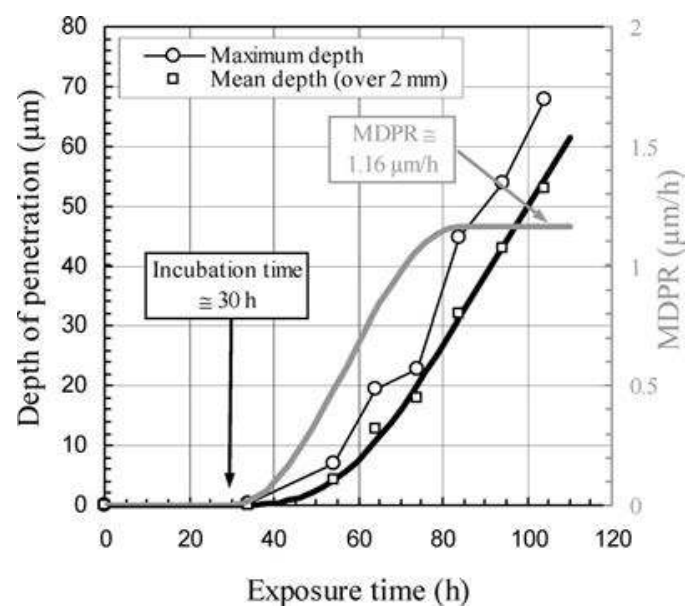

Fig. 18 Mean depth of penetration (in black, left scale) and mean depth of penetration rate (in gray, right scale) versus exposure time. The gray curve is the time derivative of the thick black curve. future comparisons with theory.

The evolution of the mean depth of penetration with exposure time presented in Fig. 18 is quite conventional. After an incubation period during which the depth of penetration remains zero, an acceleration period is observed, followed by a quasilinear increase in the depth of penetration with exposure time. The MDPR was obtained by derivation with respect to time. For further validation, special attention is paid to the constant value of MDPR during the steady-state regime of erosion. The existence of a steady-state regime characterized by a linear increase in depth of penetration with time is a good indicator of the invariance of the erosive potential of the cavitating flow, confirmed by pitting tests. The duration of this steady-state period and the evolution of mass loss beyond $104 \mathrm{~h}$ were not investigated.

\section{Analysis of Experimental Results}

4.1 Estimation of Erosive Potential. The first step in the prediction procedure is the estimation of the erosive potential of the cavitating flow in terms of the three integral parameters considered in the present work: mean impact load $\bar{\sigma}$, mean area of impact load $\bar{S}$ (or equivalent mean diameter $\bar{D}$ ), and pitting rate $N$. For each pit, the impact load responsible for it is deduced from the measurement of its maximum depth. Let us first consider the case of an impact load $\sigma$ smaller than the ultimate strength $\sigma_{U}$ of the material. Plastic deformation occurs without any material removal, and the depth of the resulting indentation is

$$
H=\int_{x=0}^{\ell} \varepsilon(x) d x
$$

Using Eq. (3) for the strain profile $\varepsilon(x)$ inside the material, we obtain

$$
H=\frac{\varepsilon_{0} \ell}{1+\theta}
$$

and finally, by means of Eq. (7),

$$
H=\frac{\varepsilon_{U} L}{1+\theta}\left(\frac{\boldsymbol{\sigma}-\sigma_{Y}}{\sigma_{U}-\sigma_{Y}}\right)^{(1+\theta) / n \theta}
$$

This equation is used to estimate the impact load $\sigma$ from the measured depth $H$ of the indentation. It is valid for flows of moderate aggressiveness, i.e., for $\sigma<\sigma_{U}$. In the limit case of an impact load equal to the material ultimate strength $\sigma=\sigma_{U}$, the depth of the indentation is

$$
H=\frac{\varepsilon_{U} L}{1+\theta}
$$

In the case of highly erosive potential defined by $\sigma>\sigma_{U}$ (which is not the case in the present experiment), some material is removed and the depth of the removed material given by Eq. (14) has to be added to the previous depth (Eq. (22)) to obtain the actual pit depth,

$$
H=\frac{\varepsilon_{U} L}{1+\theta}+L\left[\left(\frac{\boldsymbol{\sigma}-\sigma_{Y}}{\sigma_{U}-\sigma_{Y}}\right)^{1 / n \theta}-1\right]
$$

Previous Eq. (23) is valid only for $\sigma>\sigma_{U}$.

Equations (21) and (23) are represented in Fig. 19 for stainless steel $316 \mathrm{~L}$. The limit value (22) estimated using material characteristics (2) and (4) is $15.8 \mu \mathrm{m}$. Since the maximum measured pit depth on the sample of 800 pits considered here is $4.8 \mu \mathrm{m}$ (see Fig. 12), it is concluded that all impact loads are below the material ultimate strength. The maximum load corresponding to the maximum pit depth is $777 \mathrm{MPa}$. In other words, the present cavitating flow with a velocity of $65.3 \mathrm{~m} / \mathrm{s}$ can be considered as a cavitating flow of moderate aggressiveness. The influence of flow velocity was not investigated here. It is generally reported that erosion damage increases with a relatively high power of the flow 


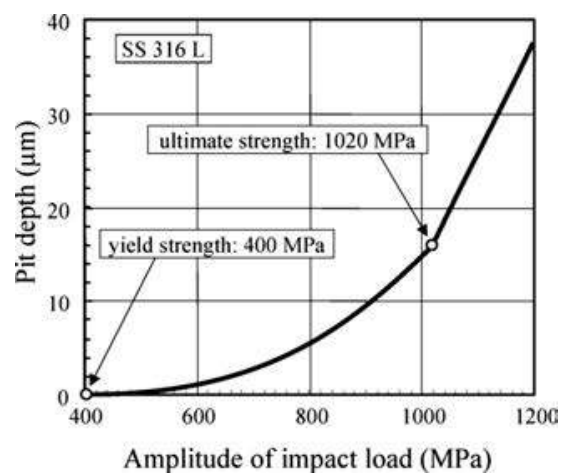

Fig. 19 Pit depth versus amplitude of impact load for stainless steel $316 \mathrm{~L}$. The two branches correspond to Eq. (21) if $\sigma<\sigma_{U}$ and Eq. (23) if $\sigma>\sigma_{U}$.

velocity of the order of 6 [31].

The previous correspondence between pit depth and impact load is systematically applied to each pit. A distribution of hydrodynamic impact loads is then obtained. In order to define a mean value representative of the whole spectrum, an averaging procedure is used based on energy arguments. From a dimensional viewpoint, the product of pit volume $V$ and impact load $\sigma$ has the units of energy. Hence, it was decided to define the mean impact load $\bar{\sigma}$ by the following averaging procedure:

$$
\bar{\sigma}=\frac{\sum V_{i} \sigma_{i}}{\sum V_{i}}
$$

where the summation is relative to all identified pits. The previous definition of mean impact load is based on the principle of conservation of energy discharged in the material. In the present case, the mean impact load determined by Eq. (24) on the sample of 797 indentations is equal to $621 \mathrm{MPa}$. This value lies between yield strength and ultimate strength, which confirms that the erosive potential of the present cavitating flow is moderate and that fatigue and work-hardening processes prevail. The mean surface of impact load $\bar{S}$ is defined by a classical averaging procedure,

$$
\bar{S}=\frac{1}{\nu} \sum S_{i}
$$

where $\nu$ is the total number of pits analyzed. As for the pitting rate $N$, it is simply the number of pits per unit time and unit surface area.

The influence of the number of pits used for the determination of flow aggressiveness was investigated for each of the three variables $\bar{\sigma}, \bar{D}$, and $N$. The analysis showed that flow aggressiveness can be considered as correctly defined by a sample of typically a few hundreds of pits (see Fig. 20 for the influence of the number of pits on MDPR and incubation time). In practice, 797 pits were considered here. For the present cavitating flow, the mean diameter of impact loads is $72 \mu \mathrm{m}$. This value is near the characteristic value of pit diameter, leading to maximum damage as deduced from Figs. 14 and 15.

4.2 Incubation Time and MDPR. Once flow aggressiveness defined by the quantities $\bar{\sigma}, \bar{S}$, and $N$ has been computed, it is possible to apply the predictive method developed in Secs. 2.5 and 2.6 and, particularly, Eqs. (12) and (15) to predict the incubation period and mass loss rate. Flow aggressiveness has been estimated from eight different surfaces similar to the one presented in Fig. 10 relative to the same pitting test but to eight different angular positions on the target at the same radius corresponding to maximum damage.

There is a non-negligible dependence of the predicted values of
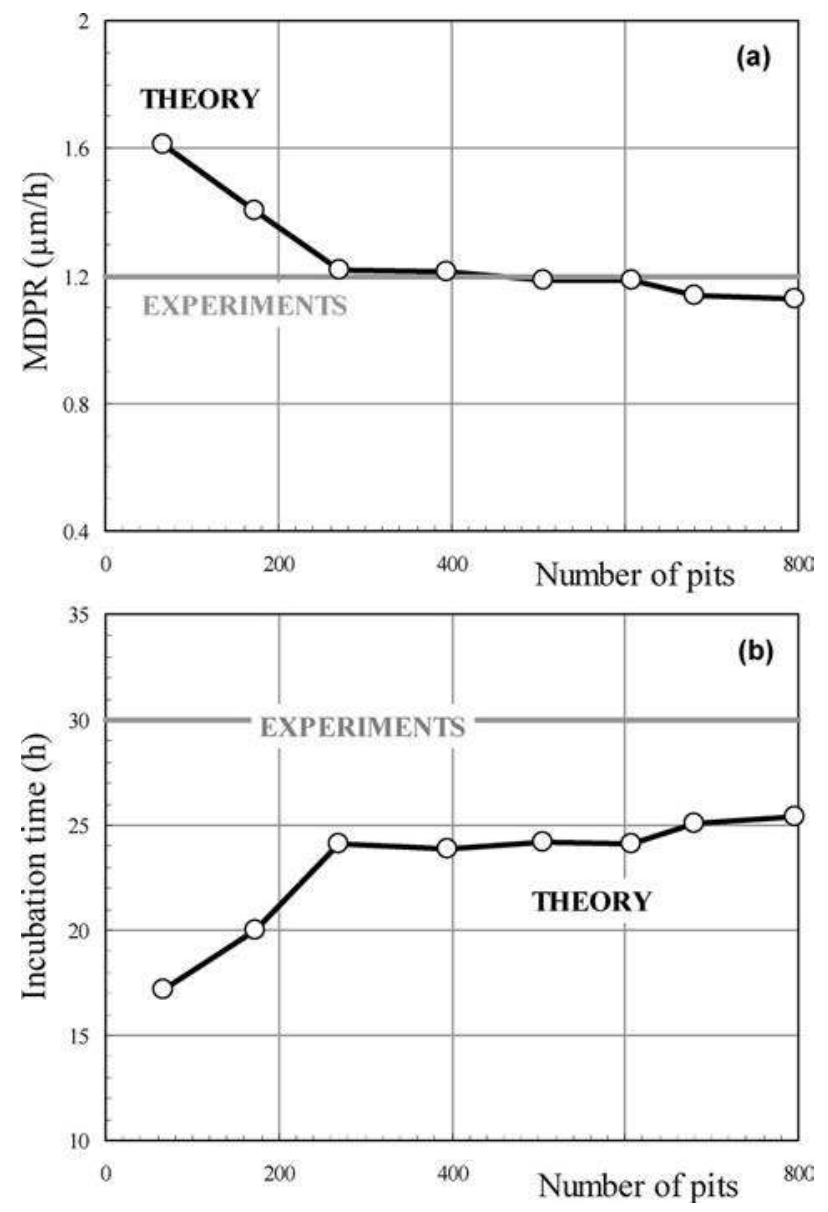

Fig. 20 Comparison between theory and experiments for (a) MDPR and $(b)$ incubation time. The theoretical prediction depends on the number of pits used to estimate the erosive potential of the cavitating flow. Prediction can be considered as stabilized above a few hundred pits.

MDPR and incubation period on the pitted surface considered to evaluate the erosive potential. Following the previous discussion on the influence of the size of the sample on the evaluation of the erosive potential of the cavitating flow, it can be expected that the values corresponding to the total number of pits analyzed here are actually good estimates of the long term damage and are suitable for a comparison with experiments. Figure 20 shows that the predicted values of MDPR $(1.1 \mu \mathrm{m} / \mathrm{h})$ and incubation time $(25.4 \mathrm{~h})$ based on the total number of pits (797 pits) are in reasonable agreement with the experimental measurements deduced from Fig. $18(1.16 \mu \mathrm{m} / \mathrm{h}$ and $30 \mathrm{~h})$.

A careful examination of the values of MDPR and incubation time predicted from the eight pitting surfaces mentioned above showed that both variables are strongly correlated. A small MDPR is associated with a long exposure time, both being the consequence of a relatively small aggressiveness. The product of the incubation time $T$ by MDPR proved to be almost constant. Using Eqs. (12) and (15) for incubation time $T$ and MDPR, respectively, and multiplying them together in order to compute the product MDPR $\times T$, it appears that the covering time $\tau$ vanishes. The only hydrodynamic parameter remaining in the product is the amplitude of impact loads $\bar{\sigma}$ (which also appears through $\varepsilon^{\prime}$ ), all other parameters being material properties, which remain unchanged for a given material. In the present case, it has been observed that the value of $\bar{\sigma}$ for the various computations is nearly constant. This explains the almost constant value of the product $\operatorname{MDPR} \times T$ in the present case. 


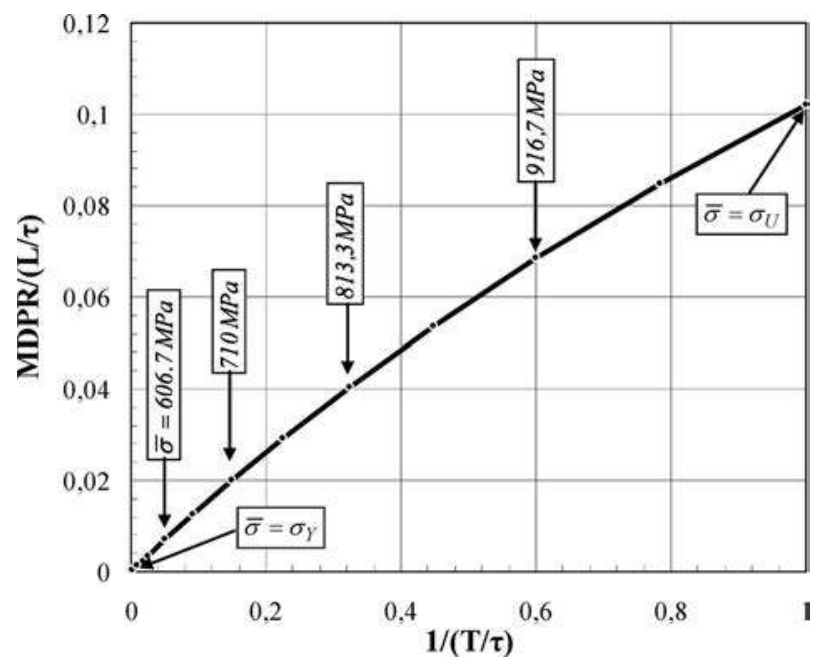

Fig. 21 Relation between nondimensional MDPR and inverse of nondimensional incubation time for stainless steel $316 \mathrm{~L}$. The curve has been obtained by varying the flow aggressiveness in terms of mean amplitude of impact load $\bar{\sigma}$.

More generally, several works have attempted to derive a relation between incubation time and MDPR. Soyama and Futakawa [32], for instance, recently showed that the incubation time can be estimated by evaluating the amount of erosion at a postincubation point. Hammitt [13] and Zhou and Hammitt [23] suggested the following form for this correlation:

$$
\frac{1}{\mathrm{MDPR}}=k T^{\alpha}
$$

where parameter $k$ should remain constant for a given material and type of test. Available estimates of exponent $\alpha$ from both cavitation and liquid impact data indicate $0.6<\alpha<1$ [13]. Using the present model, nondimensional MDPR was plotted in Fig. 21 for SS $316 \mathrm{~L}$ versus the inverse of the nondimensional incubation time to further analyze the relation between both parameters. Each point on the curve corresponds to a given value of flow aggressiveness in terms of impact load $\bar{\sigma}$. The whole curve was obtained by varying $\bar{\sigma}$ between the material yield strength and its ultimate strength, the latter being the maximum value for which the incubation time still has meaning. It then refers to a quite large range in flow aggressiveness. From the examination of Fig. 21, it can be concluded that the present model predicts an almost linear dependence between MDPR and the inverse of the incubation period in the whole range of variation in flow aggressiveness.

Let us observe that the present simplified model fails at predicting the acceleration period clearly visible in Fig. 18. This is due to the noninclusion in the model of the statistical nature of impact loads in terms of amplitude, size, and space distribution. As a consequence, strain is assumed uniform on the material surface at any time, and ultimate strain and subsequent mass loss are reached at the same time at any point of the material surface. Then, there is no distinction between the end of the incubation period and the beginning of the steady-state erosion period so that a steplike behavior for MDPR versus exposure time is found. As shown by Berchiche et al. [25], a more realistic variation with an acceleration period would be obtained by considering the whole spectrum of impact loads (and not only average values) and assuming that impact loads are randomly distributed in space. If so, ultimate strain progressively gains the whole material surface depending on the amplitude of successive impact loads and their possible partial overlapping. This results in a transitional regime between incubation and steady-state erosion characterized by a gradual increase in the erosion rate, as shown experimentally. However, in the present paper, we have deliberately chosen to ignore the random distribution in space, size, and amplitude of impact loads in order to be able to derive analytical relationships and more easily point out the key parameters of the damage process, which was our primary objective. ${ }^{1}$

A final remark concerns the evaluation of the influence of the threshold chosen for the analysis of pitting tests on the prediction of the incubation period and erosion rate. An excessive value of the threshold obviously tends to underestimate the number and size of pits, whereas a too small value will overestimate them and even lead neighboring pits to merge. It is difficult to derive an objective criterion for the choice of this threshold. Here, the choice is made on the basis of a qualitative comparison of the original image to the binary one obtained after applying the threshold. From Fig. 22, it is clear that the two extreme thresholds $0.8 \mu \mathrm{m}$ and $0.3 \mu \mathrm{m}$ considered here are not acceptable, whereas the threshold $0.5 \mu \mathrm{m}$ can be considered to give a relatively satisfactory description of the pitted surface. It is of major concern to observe that if the number and size of pits depend on the threshold, pit depths, which are measured from the original virgin surface and not from the threshold level (see Fig. 11), are unchanged, as well as the subsequent amplitudes of impact loads. The influence of the threshold on the predicted incubation time and erosion rate is presented in Fig. 23, together with the experimental measurements. The higher the threshold, the higher the incubation time and the smaller the erosion rate. Although the predicted values depend on the precise value of the threshold, Fig. 23 shows that in the range of $0.4-0.6 \mu \mathrm{m}$, which is considered acceptable from visualizations of Fig. 22, they remain of the same order of magnitude as in experiments.

\section{Concluding Remarks}

The present paper focuses on the prediction of incubation time and erosion rate of ductile materials exposed to a cavitating flow. From a dimensional viewpoint, the erosion rate measured in terms of the MDPR has the units of a characteristic length divided by a characteristic time. The present work suggests that

(i) The relevant time scale is the covering time $\tau$, i.e., the time necessary for the surface to be exactly covered by the hydrodynamic impacts. This time depends on the impact rate and the size of the loaded areas. It is a feature of the cavitating flow in so far as it depends mainly on hydrodynamic aspects (such as bubble production rate and bubble size).

(ii) The relevant length scale for ductile materials is the thickness of the hardened layer $L$. It can be determined from microhardness measurements on cross sections of eroded specimens. This is essentially a material characteristic, although it probably integrates to some extent the very specific type of loading due to bubble collapses since it is determined on specimens actually eroded by cavitation.

MDPR can be estimated by the ratio $L / \tau$ with a multiplicative factor, which depends principally on flow aggressiveness in terms of the mean amplitude of the hydrodynamic impact loads. It is zero in the limit case of impact loads equal to the yield strength and progressively increases with flow aggressiveness. An explicit formulation is proposed to estimate this dependency.

As for the incubation time, the present approach shows that it is proportional to the covering time and that the ratio is strongly dependent on the flow aggressiveness. For impact loads close to the material ultimate strength, the incubation time matches the covering time. When the impact load approaches the material yield strength, i.e., for flows of relatively small aggressiveness, the incubation time increases considerably and can be several or-

${ }^{1}$ This difference in shape between the theoretical and experimental evolutions of MDPR with exposure time may be the reason for a poorer comparison between theory and experiment for the incubation period compared with MDPR (see Fig. 19). 


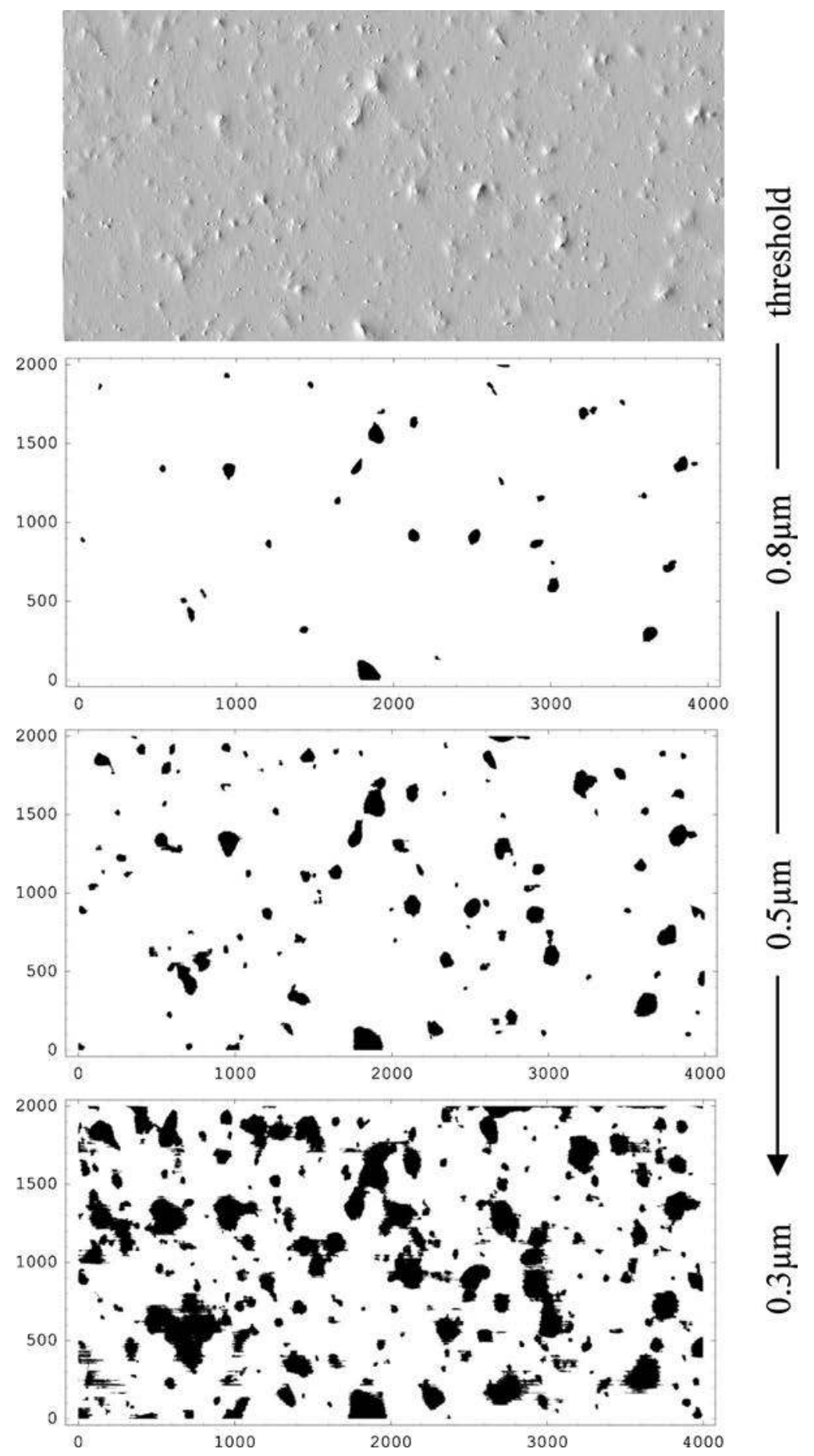

Fig. 22 Influence of depth threshold on the treatment of a pitting test (same surface as in Fig. 10)

ders of magnitude larger than the covering time. The model proposed here accounts for this variation. It also allows us to predict the relationship between MDPR and the inverse of incubation time, which appears to be almost linear in the whole range of flow aggressiveness.

The model is supported by an experimental investigation in which erosion is produced by a cavitating flow in a radial divergent. In the absence of any reliable method of estimation of flow aggressiveness from global hydrodynamic parameters (such as cavitation number and flow rate), pitting tests are used to quantify the erosive potential of the cavitating flow. Hydrodynamic impact loads are deduced from the measurement of the maximum pit depth by means of the same model of response of the material as the one used for mass loss prediction. As for the rate and surface area of the impact loads, they are deduced from the pitting rate and pit size. By introducing such an estimate of the erosive potential of the cavitating flow into the model, it has been possible to predict the incubation period and mass loss rate under steady-state conditions. Predictions are compared with experimental values obtained from mass loss experiments. The tested material is stainless steel $316 \mathrm{~L}$, which is known to exhibit high work hardening. 


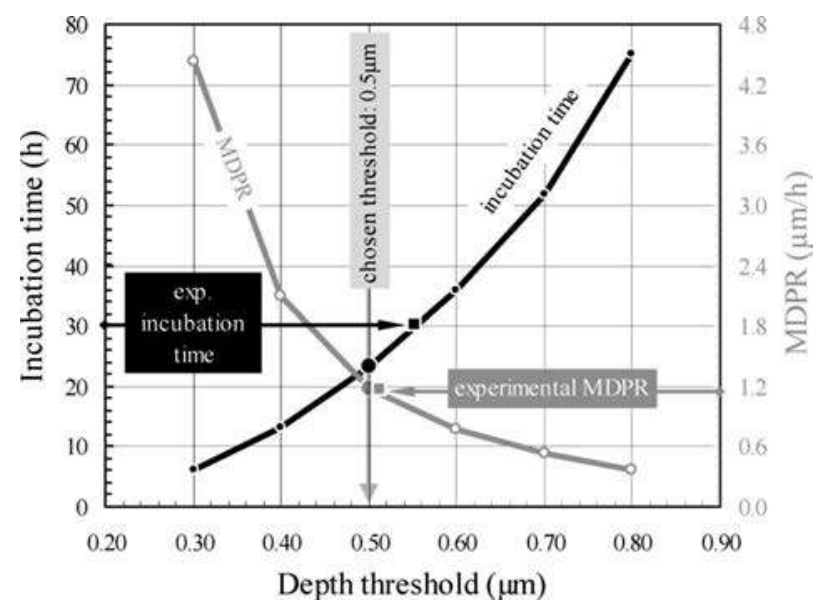

Fig. 23 Influence of depth threshold on predicted incubation period and erosion rate

On the whole, the agreement between predicted and measured values of the incubation time and MDPR is satisfactory.

The present approach is based on an elementary modeling of the flow aggressiveness. It consists in approximating the whole impact load spectrum by mean values of both the impact loads and the impacted areas. This results in a steplike variation in the erosion rate versus the exposure time with no mass loss before the incubation time and a constant steady-state erosion beyond. To account for the acceleration period, which actually exists between the incubation and the steady-state periods, as shown by experiments, it is necessary to take into account the whole distribution of impact loads in size and amplitude and also the spatially random nature of the hydrodynamic impacts. This can easily be done in the framework of the same phenomenological analysis but requires the development of a numerical procedure. The objective of the present work based on a simplified characterization of the erosive potential of a cavitating flow was essentially to contribute to elucidating the erosion mechanism of ductile materials, point out the major parameters that control damage, and propose a deterministic approach of the erosion process. Although it is still under development, such a technique of prediction of cavitation erosion can be considered as a future alternative to more conventional techniques based on correlations with material properties such as Vickers hardness, ultimate resilience, and fatigue tests (see, e.g., Refs. 13 and 33-35).

\section{Acknowledgment}

The present research has been conducted in the framework of the European project "PREVERO" under Contract No. ENK6-CT2002-00605. The author is particularly grateful to AVL, coordinator of the project, and all other partners of the consortium for fruitful discussions. The author would also like to acknowledge the essential help provided by J.C. Jay, Y. Lecoffre, J.M. Michel, and M. Riondet for the design of facility and experiments.

\section{Nomenclature}

$H=$ maximum pit depth $(\mathrm{m})$

$K=$ constant in stress/strain relationship (1) $(\mathrm{Pa})$

$\ell=$ thickness of the hardened layer for partial hardening $(\mathrm{m})$

$L=$ thickness of the hardened layer for complete hardening $(\mathrm{m})$

MDPR $=$ mean depth of penetration rate $(\mathrm{m} / \mathrm{s})$

$n=$ exponent in stress/strain relationship (1)

$N=$ impact rate per unit surface area (impacts $/ \mathrm{m}^{2} / \mathrm{s}$ )

$p_{d}=$ downstream pressure in test section $(\mathrm{Pa})$

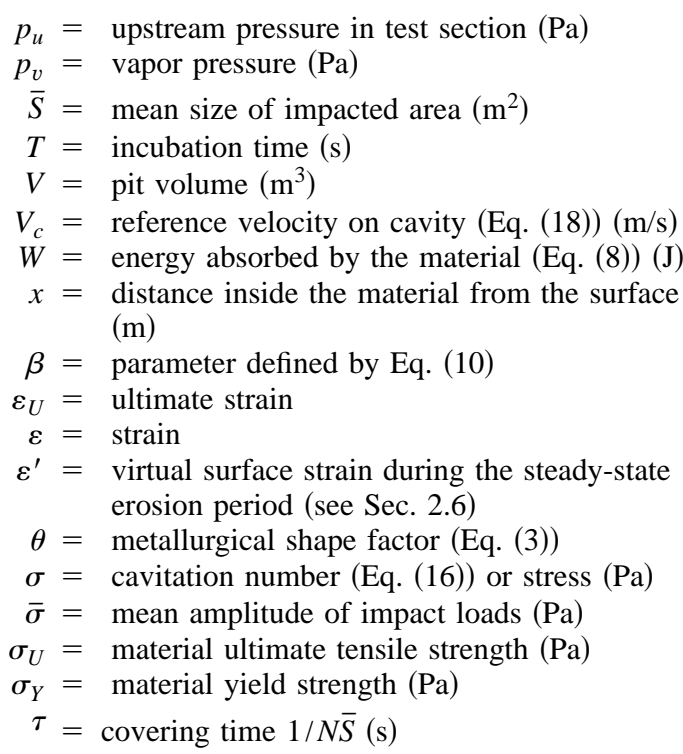

\section{References}

[1] Pereira, F., Avellan, F., and Dupont, Ph., 1998, "Prediction of Cavitation Erosion: An Energy Approach,” ASME J. Fluids Eng., 120, pp. 719-727.

[2] Lecoffre, Y., Marcoz, J., Franc, J. P., and Michel, J. M., 1985, "Tentative Procedure for Scaling Cavitation Damage," Proceedings of the Joint ASCE/ ASME Mechanics Conference on "Cavitation in Hydraulic Structures and Turbomachinery," Albuquerque, NM, Jun. 24-26, R. E. A. Arndt and D. R. Webb, eds., FED-Vol. 25, pp. 1-11.

[3] Kato, H., 1975, “A Consideration on Scaling Laws of Cavitation Erosion,” Int. Shipbuild. Prog., 22(253), pp. 305-327.

[4] Kato, H., Konno, A., Maeda, M., and Yamaguchi, H., 1996, "Possibility of Quantitative Prediction of Cavitation Erosion Without Model Test," ASME J. Fluids Eng., 118, pp. 582-588.

[5] Maeda, M., Yamaguchi, H., and Kato, H., 1991, "Laser Holography Measurement of Bubble Population in Cavitation Cloud on a Foil Section" First ASME-JSME Fluids Engineering Conference, Portland, OR, Jun. 23-27, H. Kato and O. Furuya, eds., FED-Vol. 116, pp. 67-75.

[6] Tomita, Y., and Shima, A., 1986, "Mechanism of Impulsive Pressure Generation and Damage Pit Formation by Bubble Collapse," J. Fluid Mech., 169, pp. $535-564$.

[7] Vogel, A., Lauterborn, W., and Timm, R., 1989, "Optical and Acoustic Investigations of the Dynamics of Laser-Produced Cavitation Bubbles Near a Solid Boundary," J. Fluid Mech., 206, pp. 299-338.

[8] Philipp, A., and Lauterborn, W., 1998, "Cavitation Erosion by Single LaserProduced Bubbles," J. Fluid Mech., 361, pp. 75-116.

[9] Reisman, G. E., Wang, Y.-C., and Brennen, C. E., 1998, "Observation of Shock Waves in Cloud Cavitation," J. Fluid Mech., 355, pp. 255-283.

[10] Knapp, R. T., Daily, J. W., and Hammitt, F. G., 1970, Cavitation, McGrawHill, New York

[11] Stinebring, D. R., Holl, J. W., and Arndt, R. E. A., 1980, "Two Aspects of Cavitation Damage in the Incubation Zone: Scaling by Energy Considerations and Leading Edge Damage," ASME J. Fluids Eng., 102, pp. 481-485.

[12] Fortes Patella, R., Reboud, J. L., and Archer, A., 2000, "Cavitation Damage Measurement by 3D Laser Profilometry," Wear, 246, pp. 59-67.

[13] Hammitt, F. G., 1979, "Cavitation Erosion: The State of the Art and Predicting Capability," Appl. Mech. Rev., 32(6), pp. 665-675.

[14] Hattori, S., Sun, B.-H., Hammitt, F. G., and Okada, T., 1985, "An Application of Bubble Collapse Pulse Height Spectra to Venturi Cavitation Erosion of 1100-O Aluminium," Wear, 103, pp. 119-131.

[15] Okada, T., Iwai, Y., Hattori, S., and Tanimura, N., 1995, "Relation Between Impact Load and the Damage Produced by Cavitation Bubble Collapse," Wear, 184, pp. 231-239.

[16] Hattori, S., Mori, H., and Okada, T., 1998, "Quantitative Evaluation of Cavitation Erosion," ASME J. Fluids Eng., 120, pp. 179-185.

[17] Knapp, R. T., 1955, "Recent Investigations of the Mechanics of Cavitation and Cavitation Damage," Trans. ASME, October, pp. 1045-1054.

[18] Knapp, R. T., 1958, "Accelerated Field Tests of Cavitation Intensity," Trans. ASME, January, pp. 91-102.

[19] Belahadji, B., Franc, J. P., and Michel, J. M., 1991, "A Statistical Analysis of Cavitation Erosion Pits," ASME J. Fluids Eng., 113, pp. 700-706.

[20] Thiruvengadam, A., 1974, "Handbook of Cavitation Erosion," Hydronautics Inc., Technical Report No. 7301-1.

[21] Karimi, A., and Martin, J. L., 1986, "Cavitation Erosion of Materials," Int. Met. Rev., 31(1), pp. 1-26.

[22] Pallabazzer, R., and Mancuso, G., 1998, "Erosive Cavitation Tests in Water Tunnel," XIX Symposium AIRH, pp. 500-509.

[23] Zhou, Y. K., and Hammitt, F. G., 1983, "Cavitation Erosion Incubation Period," Wear, 86, pp. 299-313. 
[24] Karimi, A., and Leo, W. R., 1987, "Phenomenological Model for Cavitation Rate Computation," Mater. Sci. Eng., 95, pp. 1-14.

[25] Berchiche, N., Franc, J. P., and Michel, J. M., 2002, "A Cavitation Erosion Model for Ductile Materials,” ASME J. Fluids Eng., 124, pp. 601-606.

[26] Ball, A., 1983, "On the Importance of Work Hardening in the Design of Wear-Resistant Materials," Wear, 91, pp. 201-207.

[27] Li, S., Zhang, Y., and Hammitt, F. G., 1986, "Characteristics of Cavitation Bubble Collapse Pulses, Associated Pressure Fluctuations, and Flow Noise,” J. Hydraul. Res., 24(2), pp. 109-122.

[28] Nguyen, M., Franc, J. P., and Michel, J. M., 1987, "On Correlating Pitting Rate and Pressure Peak Measurements in Cavitating Flows," J. W. Holl and M. L. Billet, eds., Proceedings of the ASME International Symposium on Cavitation Research Facilities and Techniques, ASME, Boston, Dec. 13-18, FED-Vol. 57, pp. 207-216.

[29] Fry, S. A., 1989, “The Damage Capacity of Cavitating Flow From Pulse Height Analysis," ASME J. Fluids Eng., 111, pp. 502-509.
[30] Momma, T., and Lichtarowicz, A., 1995, “A Study of Pressures and Erosion Produced by Collapsing Cavitation," Wear, 186-187, pp. 425-436.

[31] Franc, J. P., and Michel, J. M., 2004, Fundamentals of Cavitation, Kluwer Academic, Dordrecht

[32] Soyama, Y., and Futakawa, M., 2004, "Estimation of Incubation Time of Cavitation Erosion for Various Cavitating Conditions," Tribol. Lett., 17(1), pp. 27-30.

[33] Hattori, S., Ishikura, R., and Zhang, Q., 2004, "Construction of Database on Cavitation Erosion and Analyses of Carbon Steel Data," Wear, 257(9-10), pp. $1022-1029$.

[34] Hattori, S., Maeda, K., and Zhang, Q., 2004, "Formulation of Cavitation Erosion Behavior Based on Logistic Analysis," Wear, 257(9-10), pp. 1064-1070.

[35] Bedkowski, W., Gasiak, G., Lachowicz, C., Lichtarowicz, A., Lagoda, T., and Macha, E., 1999, "Relations Between Cavitation Erosion Resistance of Materials and Their Fatigue Strength Under Random Loading," Wear, 230, pp. 201-209. 